\title{
Cuidados domiciliares ao idoso Que sofreu Acidente Vascular Cerebral
}

\author{
Home care to the elderly who had stroke
}

Atención domiciliaria al anciano que sufrió Accidente Cerebrovascular

\section{Larissa Chaves Pedreira', Regina Lúcia Mendonça Lopes'}

'Universidade Federal da Bahia. Curso de Enfermagem. Núcleo de Estudos e Pesquisas sobre o Idoso. Salvador, BA

Submissão: 14/07/2009 Aprovação: 10/07/2010

\section{RESUMO}

O objetivo foi identificar a produção do conhecimento sobre acidente vascular cerebral no idoso cuidado no domićlio. Estudo bibliográfico cuja coleta foi através dos resumos datados de 1997 a 2007, nos bancos de dados LILACS e SciELO. Utilizou-se como descritores: assistência domiciliar, idoso e acidente cerebrovascular. Foram encontradas 52 referências no LILACS, nove na SciELO Brasil e três no SciELO Cuba. A maioria dos trabalhos foi realizada em 2000. Quanto ao método, pesquisas com abordagem Qualitativa predominaram, Sendo o tema principal relacionado ao cuidador, aspectos clínicos e epidemiológicos da doença. Observou-se Que esse conhecimento está sendo solidificado no Brasil e temas importantes relacionados à pessoa sujeita dos cuidados e a violência domiciliar ao idoso são pouco explorados.

Descritores: Idoso; Assistência domiciliar; Acidente cerebrovascular; Literatura de revisão.

\section{ABSTRACT}

The purpose was to Identify the knowledge production about the stroke in elderly under home care. Bibliographic research whose data were collected though the abstracts from 1997 to 2007, contained in LILACS and SciELO atabases. The following key words were used: home assistance, aged people and cerebrovascular accident. Fifty-two references were found in the LILACS database, nine in the SciELO Brazil, and three in the SciELO Cuba. Most of the researches were carried out in 2000. Regarding the method, Qualitative method predominace were observed, and central theme is related to the care giver, as well as to the clinical and epidemiologic aspects of the disease. It was observed that this knowledge is still established in Brazil, and the themes related to the person submitted to home care and violence to the aged are still little explored.

Key words: Aged; Home nursing; Cerebrovascular accident; Review literature.

\section{RESUMEN}

El objetivo fue Identificar la producción del conocimiento sobre accidente vascular cerebral en el anciano cuidado en el domicílio. Estudio bibliográfico, cuantitativo, cuya colecta de datos fue realizada en julio de 2007, a través de los resúmenes fechados desde 1997 a 2007, contenidos en los bancos de datos LILACS y SciELO, de países de América Latina y Caribe. Para la colecta fueron utilizados los descriptores: Atención Domiciliaria de Salud, anciano y accidente cerebrovascular. Fueron encontradas 52 referencias en el LILACS, nueve en el SciELO Brasil y trés en el SciELO Cuba. La mayoria de los trabajos fue realizada en 2000, con predominancia en el método cualitativo, y el tema central es relacionado al cuidador, a los aspectos clínicos y epidemiológicos de la enfermedad. Se observó que en Brasil, ese conocimiento aú está siendo solidificado y los temas relacionados a la persona sujeta a la atención y a la violencia domiciliaria al anciano todavia son poco explorados.

Descriptores: Anciano; Atención domiciliaria de salud; Accidente cerebrovascular; Literatura de revisión 


\section{INTRODUÇÃO}

A extensão do acidente vascular cerebral (AVC), hoje considerado como a terceira causa de morte na população adulta/idosa, tem levado muitos de seus acometidos à necessidade de cuidados, muitos destes só possíveis no domicílio.

Atualmente, o AVC é o maior causador de incapacidade funcional no ocidente ${ }^{(I)}$, provocando, principalmente em idosos, alterações na capacidade de desempenhar atividades cotidianas. Essas alterações podem ser passageiras ou não, de acordo com a região acometida, o nível da lesão e a capacidade individual de recuperação.

Nesse contexto, é sabido Que o número de idosos vem aumentando e, de acordo com os demógrafos, a previsão é de Que em 2020 existam 34 milhões de idosos brasileiros, correspondendo à sexta população mais velha do planeta.

Por conta desta incapacidade funcional, o AVC pode gerar uma diminuição da Qualidade de vida com um impacto na vida cotidiana, destarte da pessoa idosa. Em termos familiares, muitas vezes, advêm conflitos envolvendo muitos de seus membros, por conta das atribuições do cuidar de um familiar com sequelas.

Entretanto, a redução de custos da assistência hospitalar e institucional a idosos incapacitados tem levado muitos países, como o Brasil, a indicar a sua permanência em casa sob os cuidados da família ${ }^{(2)}$

Sobre isso, Caldas ${ }^{(3)}$ faz uma crítica apontando que, no Brasil, apesar da Política Nacional de Saúde do Idoso, não existe um programa de governo direcionado para esta população com dependência. Assim, com a falência crescente do sistema previdenciário, os cuidados prestados no domicílio junto à família vêm se tornando à única opção disponível para o idoso dependente.

No Brasil, algumas discussões têm sido realizadas sobre esse tema desde a década de 1980, Quando os idosos, já se fazendo presentes de forma significativa, passaram a ser percebidos pelo governo demandando políticas públicas.

Em fevereiro de 2006, surge a Portaria n³99/GM, Que aborda as Diretrizes do Pacto pela Saúde, contemplando o Pacto pela Vida, sendo a saúde do idoso uma das seis prioridades pactuadas entre as três esferas governamentais ${ }^{(4)}$. Aqui, são apresentadas ações Que visam à implementação de algumas das diretrizes da Política Nacional de Atenção à Saúde do Idoso de 1994(5).

Entre essas, estão as Que pretendem responder ao número insuficiente de serviços de cuidado domiciliar ao idoso frágil. Sendo a família, via de regra, a executora de cuidados, evidencia-se a necessidade de se estabelecer um suporte Qualificado e constante aos responsáveis por estes, tendo a atenção básica por meio da Estratégia Saúde da Família um papel fundamental.

Em 19 de outubro de 2006, surge também a Política Nacional de Saúde da Pessoa Idosa, Que tem, dentre seus objetivos, identificar o nível de dependência do idoso e acompanhar de forma diferenciada cada situação(6).

Apesar das demandas expostas acima, no Brasil, a temática do cuidador e seus cuidados domiciliares a idosos vitimados por AVC é um tema ainda pouco abordado, sendo seu conhecimento essencialmente internacional ${ }^{(7)}$.

Esse trabalho tem como objetivo identificar a produção do conhecimento sobre acidente vascular cerebral no idoso cuidado no domicílio. Pretende-se com esse estudo, oferecer subsídios Que direcionem o desenvolvimento de estudos na área de assistência domiciliar.

\section{METODOLOGIA}

Pesquisa bibliográfica cujos dados foram coletados nas bases de dados Latin American Literature in Health Sciences (LILACS) e Scientific Electronic Library Online (SCIELO), dos países da América Latina e Caribe disponíveis (Argentina, Colômbia, Cuba, Venezuela, Peru, Costa Rica, Uruguai, Chile e México), a partir dos descritores: idoso, cuidado domiciliar e acidente cerebrovascular. As bases foram acessadas em julho de 2007.

Foram considerados os trabalhos publicados a partir de 1997 até 2007, Que tivessem pelo menos dois dos descritores utilizados nas palavras-chave, ou no resumo, já Que muitos trabalhos diziam sobre o tema no resumo e no título, mas não traziam a palavra como descritor. Assim, foram encontrados 64 referências de artigos de revista, dissertações, teses e documentos. Do material encontrado, foram descartados materiais sem data, bem como capítulos de livro.

A coleta dos dados foi feita com a leitura dos resumos e o preenchimento de um formulário Que continha as seguintes variáveis independentes: ano de publicação, local de publicação, metodologia aplicada, área temática e veículo de publicação.

Os dados foram organizados estatisticamente e apresentados em números e na forma de Quadros. A análise dos dados deu-se a partir de teóricos da literatura pertinente.

\section{RESULTADOS}

O número de trabalhos encontrados, de acordo com os critérios descritos na metodologia foram nove na base do SciELO Brasil, três na base do SciELO Cuba e 52 na base do LILACS. Na base de dados SciELO dos outros países da América Latina e Caribe, não foram encontradas referências com os descritores selecionados.

No Brasil, a maioria dos trabalhos está na Região Sudoeste, sendo que este país supera os outros da América Latina e Caribe em relação à produção na área.

Quanto à metodologia, 17 trabalhos utilizaram a metodologia Quantitativa, 42 a Qualitativa, dois a Quanti-Qualitativa e três trabalhos não tiveram a metodologia registrada nos resumos.

Os temas foram agrupados de acordo com o foco da pesquisa identificado, sendo que os estudos caracterizados como clínicos/ epidemiológicos, se referem aqueles relacionados a estudos epidemiológicos e/ou trazendo dados clínicos, de Qualeuer natureza da patologia ou do atendimento. Os temas agrupados em cuidador se referem aos que abordam aspectos das demandas deste, assim como seu processo de formação. Aqueles agrupados em pessoa cuidada, dizem respeito à pessoa Que recebe o cuidado em relação às demandas psicológicas, sociais e existenciais. Os agrupados em $\mathrm{AD}$, se referem aos aspectos históricos, políticos, econômicos e gerenciais e, finalmente, os agrupados em relação família/ profissional/pessoa cuidada, são atinentes aos aspectos envolvidos na relação interpessoal.

Foram encontrados no total 19 trabalhos voltados para a pesquisa clínico-epidemiológica, dos Quais 14 da base LILACS, 
três da SciELO Brasil e dois da SciELO Cuba; 21 trabalhos na temática cuidadores, com três da SciELO Brasil, um da SciELO Cuba e 17 da LILACS. Sobre o tema pessoa cuidada, foram encontrados 08 trabalhos, com dois da SciELO Brasil e seis da LILACS. O tema relação família/profissional/pessoa cuidada, apareceu em sete trabalhos, sendo todos da base LILACS e, se referindo a violência doméstica, só foi encontrado um trabalho nesta mesma base de dados.

Quanto o veículo de publicação, foram encontrados 55 trabalhos publicados em revistas científicas, e nove trabalhos publicados sob a forma de dissertações, teses ou documentos.

\section{DISCUSSÃO}

A expressiva Quantidade de publicação no LILACS em relação ao SciELO se deve, principalmente, pelo primeiro conter, além de artigos, resumos de teses, dissertações, capítulos de livros e documentos, enQuanto Que o segundo possui indexações apenas de revistas.

A produção do conhecimento na área no Brasil é expressivamente maior Que nos outros países da América Latina. Uma investigação sobre a pesquisa em enfermagem na América Latina entre 2000 e 2006, demonstrou Que o Brasil está em primeiro lugar na produção científica, seguido por México, Chile, Argentina e Perú(9).

Em relação ao ano de publicação do material, observa-se um aumento da produção sobre o tema a partir da década de 1990 , havendo, porém, uma maior produção no final desta e a partir do Século XXI. Certamente, esse crescimento vem acompanhando a acelerada mudança demográfica e os programas de proteção à saúde do idoso no Brasil implantados, principalmente, a partir de 1994. Outros estudos bibliográficos também mostraram essa tendência com aumento da produção a partir de $2000^{(10-11)}$

No Que se refere aos temas, observou-se um predomínio de trabalhos com abordagens referentes à demanda do cuidador no seu cotidiano de cuidar do idoso no domicílio.

Nesse sentido, a produção do conhecimento acompanha as prioridades sanitárias atuais, onde Questões relacionadas ao envelhecimento e ao papel do cuidador tem merecido destaque.

Com a deficiência do sistema formal de apoio, muitas vezes pelo seu alto custo, muitos países, dentre eles o Brasil, têm recomendado em seus programas, a permanência do idoso incapacitado no domićlio sob o cuidado de familiares. Contudo, nessa prática, geralmente os cuidadores recebem apoio apenas de outros familiares, amigos e vizinhos, ou seja, do sistema informal, gerando grandes necessidades para essa população.

As demandas das ciências da saúde na América Latina e Caribe derivam assim de características fundamentais relacionadas à situação de saúde destes países, com rápidas mudanças demográficas e epidemiológicas pelo envelhecimento da população, aumento da urbanização e da violência, declínio da mortalidade e fecundidade ${ }^{(12)}$.

Dessa forma, no Brasil, temas emergentes como doenças crônicas não transmissíveis, saúde do idoso e assistência domiciliar, passando a fazer parte da agenda do governo, foram também inseridos como prioritários em linhas de órgãos de fomento a pesquisa, Organização Mundial de Saúde e Organização PanAmericana de Saúde.
Esses temas têm sido também encontrados como foco de interesse de vários grupos de pesQuisa nacionais. Um trabalho sobre pesquisadores, temas e tendências nas pesquisas sobre envelhecimento no Brasil, demonstra Que, das linhas de pesquisa relativas a esse tema, as mais encontradas se referem aos estudos das doenças crônicas não transmissíveis, as Que se referem aos aspectos da assistência domiciliar, ficaram em Quarto lugar e em oitavo aquelas atinentes à epidemiologia ${ }^{(13)}$.

Alguns temas ainda são pouco abordados como os referentes ao sentido ou ao bem estar da pessoa a ser cuidada. Observam-se também poucos trabalhos relacionados à violência doméstica e a assistência de enfermagem prestada nessa modalidade de atendimento. Acredita-se Que as lacunas existentes sobre esses temas, ocorrem pelas grandes demandas atuais dos cuidadores Que, se não atendidas, irão refletir diretamente no bem estar do idoso.

Sobre a violência, autores colocam Que estar dependente do cuidado de outras pessoas, é um fator de risco, e Que ela ocorre com uma frequência bem maior do Que imaginado em pessoas idosas, e em todas as classes sociais ${ }^{(14)}$. Dessa forma, acredita-se Que ser a violência familiar um tema pouco investigado, provavelmente pelas dificuldades de se entrar no âmbito destas Questões a nível domiciliar.

Quanto à abordagem metodológica, há um predomínio da abordagem Qualitativa sobre a Quantitativa e a Quanti-Qualitativa. Sobre isso, é demonstrado Que até a década de 1990, estudos de abordagem clínica e epidemiológica ocuparam um grande percentual nas pesquisas com seres humanos ${ }^{(13)}$. Contudo, demandas sociais, epistemológicas e filosóficas vêm mudando esse Quadro. Entre estas, o surgimento recente, no campo da saúde, de diversas propostas para sua renovação/reconstrução, pautada na integralidade, humanização, promoção da saúde e a busca da subjetividade e intersubjetividade humana ${ }^{(15)}$.

Em relação ao local de publicação, observa-se no Brasil uma predominância dos trabalhos na Região Sudeste com 41 publicações, em relação muito distinta das demais regiões. Esse dado é compreensível pelo maior número de revistas científicas, universidades e, conseQuentemente, maior concentração e fixação de pesquisadores(as) na citada região. Além disso, é no eixo RioSão Paulo Que encontra o maior número de grupos de pesQuisa sobre envelhecimento humano, destacando-se Que 76 grupos estão nos Estados de São Paulo e Rio de Janeiro, e apenas 50 grupos no restante do País ${ }^{(13)}$. Essa predominância, também foi encontrada em um outro estudo bibliográfico sobre cuidadores de idosos ${ }^{(11)}$.

Quanto ao veículo de publicação, há uma predominância por artigos de revistas científicas, o Que é algo favorável, pois difunde o conhecimento de forma rápida, sendo mais acessível, principalmente, se tais periódicos forem indexados em grandes bases de dados.

Contudo, mesmo com a colocação acima, observa-se Que na América Latina e Caribe ainda há uma grande distância entre a produção do conhecimento científico e a sua disseminação e aplicação. O resultado disso é o isolamento da pesquisa na comunidade científica, ficando este distante do resto da sociedade ${ }^{(12)}$.

A produção e disseminação da pesquisa científica, são importantes para enriQuecer o conhecimento, auxiliando na melhoria 
das práticas de saúde e na formulação e implementação de políticas públicas.

\section{CONCLUSÕES}

O trabalho teve como objetivo identificar a produção do conhecimento sobre acidente vascular cerebral no idoso cuidado no domicílio. Observou-se Que esse conhecimento ainda está sendo solidificado no Brasil, e que aqui ele se iniciou a partir da década de 1990.

Em relação aos outros países da América Latina e Caribe, a produção nesse sentido ainda é peeuena, apesar de estas nações possuírem demandas econômicas e sociais parecidas com as do Brasil.
Entre os trabalhos verificados, houve uma predominância pelas temáticas referentes aos estudos clínicos, epidemiológicos e estudos envolvendo o cuidador, ficando temas importantes como àQueles relacionados à pessoa sujeita dos cuidados e a violência domiciliar com poucas referências. Quanto à metodologia, houve uma preferência pelas pesQuisas Qualitativas.

Cabe ressaltar Que alguns trabalhos também importantes, apesar de poucos, ficaram fora da presente coleta por não apresentarem, em seus descritores, as palavras selecionadas.

Observa-se, assim, a necessidade de se divulgar melhor o conhecimento científico e de se ampliar, posteriormente, o olhar dessa pesquisa para outros bancos de dados internacionais, a fim de se ter à dimensão dessa temática em âmbito mundial.

\section{REFERÊNCIAS}

1. André C. Manual de AVC. Rio de Janeiro: Revinter; 1999.

2. Karsch UM. Idosos dependentes: famílias e cuidadores. Cad Saúde Pública 2003; 19(3): 861-6.

3. Caldas CP. Envelhecimento com dependência: responsabilidades e demandas da família. Cad Saúde Pública 2003; 19(3): 733-81.

4. Ministério da Saúde (BR). Agência Nacional de Vigilância Sanitária. Portaria no 399, de 22 de fevereiro de 2006. Divulga o Pacto pela Saúde 2006 - Consolidação do SUS e aprova as Diretrizes Operacionais do Referido Pacto. Diário Oficial União 2006; fev 23: 1.

5. Mlnistério da Saúde (BR). Lei no.8.842, de 4 de janeiro de 1994. Dispõe sobre a Política Nacional do Idoso, cria o Conselho Nacional do Idoso e dá outras providências. Diário Oficial União 1994; jan 5. Disponível em: http://www.sbgg.org. br/profissional/legislacao/lei_idoso.pdf.

6. Ministério da Saúde (BR). Portaria № 2.528 de 19 de outubro de 2006. Dispõe sobre a Política Nacional de Saúde da Pessoa Idosa. Diário Oficial União 2006 out 20. Disponível em http:/ /dtr200 I saude.gov.br/sas/PORTARIAS/Port2006/GM/GM2528.htm.

7. Bocchi SCM. Vivenciando a sobrecarga ao vir-a-ser um cuidador familiar de pessoa com acidente vascular cerebral (AVC): análise do conhecimento. Rev Latino-am Enfermagem 2004; 12(1): II5-21.
8. Pellegrini FA. Science for health: notes on the organization of scientific activity for the development of health in Latin America and the Caribbean. Rev Panam Salud Pública 2000; 7(5): 345-9.

9. Cometto MC, Piovan Mirta, GP. Aportes de los coloquios panamericanos a la investigación en enfermería: período 20002006. Texto Contexto Enferm 2008; 17(4): 720-6.

10. Rocha FCV, Brito CMS, Luz MHBA, Figueiredo MLF: Análise da produção científica sobre o idoso na REBEn. Rev Bras Enferm 2007; 60(4): 449-5I.

11. Nascimento LC, Moraes ER, Silva IC, Veloso LC, Vale ARMC. Cuidador de idosos: conhecimento disponível na base de dados LILACS. Rev Bras Enferm 2008; 6 I (4): 5 I4-7.

12. Soares, LTR. América Latina: transição epidemiológica ou retrocesso social? Acta Paul Enferm 2000; I3(1); 55-64.

13. Prado SD, Sayd ID. A pesquisa sobre envelhecimento humano no Brasil: grupos e linhas de pesquisa. Ciênc Saúde Coletiva 2004; 9(1): 57-67.

14. Menezes MR, Oliveira NA, Pedreira LC, Santos EC, Dias ML. A violência doméstica contra o idoso identificada em um programa de assistência domiciliar: estudo de caso. Rev Eletron Enferm 2008; 10(4). Disponível em: http://www.fen.ufg.br/ revista/vl0/n4/vl 0n4al 7.htm.

15. Ayres IRCM. Uma concepção hermenêutica de saúde. Physis: Rev Saúde Coletiva 2007; 17(1): 43-62. 